\title{
Hepatic Proteomic Changes and Sirt1/AMPK Signaling Activation by Oxymatrine Treatment in Rats With Non-alcoholic Steatosis
}

\author{
Hong $\mathrm{Xu}^{1 * t}$, Gao-Feng Chen ${ }^{2 \dagger}$, Yu-Shui Ma ${ }^{3}$, Hong-Wei Zhang ${ }^{4}$, Yang Zhou ${ }^{5}$, \\ Guang-Hui Liu' ${ }^{6}$, Dong-Ya Chen ${ }^{1}$, Jian Ping ${ }^{2}$, Yi-Hui Liu' ${ }^{1}$, Xin Mou ${ }^{7}$ and Da Fü
}

1 Department of Gastroenterology and Hepatology, Hangzhou Red Cross Hospital, Hangzhou, China, ${ }^{2}$ Institute of Liver Diseases, Shuguang Hospital Affiliated to Shanghai University of Traditional Chinese Medicine, Shanghai, China, ${ }^{3}$ Central Laboratory for Medical Research, Shanghai Tenth People's Hospital, Tongji University School of Medicine, Shanghai, China, ${ }^{4}$ Shanghai Institute for Advanced Immunochemical Studies, ShanghaiTech University, Shanghai, China, ${ }^{5}$ Liver Cirrhosis Section, Department of Hepatology, Shuguang Hospital Affiliated to Shanghai University of Traditional Chinese Medicine, Shanghai, China, ${ }^{6}$ Department of Endocrinology, Tongji Hospital, Tongji University School of Medicine, Shanghai, China, ${ }^{7}$ Department of Endocrinology, Hangzhou Red Cross Hospital, Hangzhou, China

\section{OPEN ACCESS}

Edited by:

Raffaele Capasso,

University of Naples Federico II, Italy

Reviewed by:

Mingfeng Xia,

Fudan University, China

lan James Martins,

Edith Cowan University, Australia

${ }^{*}$ Correspondence:

Hong Xu

hongxuhzrc@aliyun.com

$\mathrm{DaFu}$

fuda@tongji.edu.cn

${ }^{\dagger}$ These authors have contributed equally to this work

Specialty section: This article was submitted to Gastrointestinal and Hepatic Pharmacology,

a section of the journal Frontiers in Pharmacology

Received: 05 November 2019 Accepted: 14 February 2020 Published: 10 March 2020

Citation:

Xu H, Chen G-F, Ma Y-S Zhang H-W, Zhou Y, LiU G-H, Chen D-Y, Ping J, Liu Y-H, Mou $X$ and

Fu D (2020) Hepatic Proteomic Changes and Sirt1/AMPK Signaling Activation by Oxymatrine Treatment in Rats With Non-alcoholic Steatosis. Front. Pharmacol. 11:216. doi: 10.3389/fphar.2020.00216
Background: Currently, active ingredients of herbal extracts that can suppress lipid accumulation in the liver have been considered a potential treatment option for nonalcoholic fatty liver disease.

Methods: Steatosis rat model was created by high fat and high sucrose diet feeding and treated with oxymatrine (OMT). Serum biochemical parameters, liver histology and lipid profiles were examined. Hepatic differentially expressed proteins (DEPs) which were significantly changed by OMT treatment were identified by ITRAQ analysis. The expressions of representative DEPs, Sirt1 and AMPK $\alpha$ were evaluated by western blotting.

Results: OMT significantly reduced the body weight and liver weight of steatosis animals, decreased the serum levels of triglyceride and total cholesterol as well as the hepatic triglyceride and free fatty acid levels, and effectively alleviated fatty degeneration in the liver. A list of OMT-related DEPs have been screened and evaluated by bioinformatics analysis. OMT significantly decreased the expressions of L-FABP, Plin2, FASN and SCD1 and increased Sirt1 expression and AMPK $\alpha$ phosphorylation in the liver of rats with steatosis.

Conclusion: The present study has confirmed the significant efficacy of OMT for improving steatosis and revealed hepatic proteomic changes and Sirt1/AMPK signaling activation by OMT treatment in rats with steatosis.

Keywords: NAFLD, proteomic, iTRAQ, oxymatrine, sirtuin 1, AMPK

Abbreviations: ALT, alanine aminotransferase; AMPK, adenosine monophosphate- activated protein kinase; AST, aspartate aminotransferase; BP, biological processes; CC, cellular component; DEPs, differentially expressed proteins; FASN, fatty acid synthase; FDR, false discovery rate; FFA, free fatty acid; HFHSD, high fat and high sucrose diet; GO, Gene Ontology; IAA, iodoacetamide; iTRAQ, isobaric tags for relative and absolute quantification; KEGG, Kyoto Encyclopedia of Genes and Genomes; LCFAs, long-chain fatty acids; L-FABP, liver fatty acid-binding protein1; LXR, liver-X-receptor; MF, molecular function; NAFLD, non-alcoholic fatty liver disease; OMT, oxymatrine; Plin2, perilipin2; PPAR $\alpha$, peroxisome proliferator activated receptor alpha; SCD1, stearoyl-CoA desaturase 1; Sirt1, sirtuin 1; SREBF1, sterol regulatory element binding transcription factor 1; SREBP, steroid regulatory element binding protein; TC, total cholesterol; TG, triglyceride. 


\section{INTRODUCTION}

Non-alcoholic fatty liver disease (NAFLD), an important public health issue worldwide, includes a spectrum of diseases ranging from simple steatosis to a more aggressive condition of steatohepatitis. Steatohepatitis is characterized by steatosis, inflammation, and hepatocyte injury and may progress to cirrhosis. The high prevalence of NAFLD is closely associated with the dramatic rise in obesity, diabetes mellitus and dyslipidemia. It has been reported that NAFLD affects approximately $60-90 \%$ of obese individuals, up to $70 \%$ of diabetics and $27-92 \%$ of patients with hyperlipidemia (Pappachan et al., 2017; Younossi et al., 2018). NAFLD is currently considered an independent predictor of long-time adverse cardiovascular events (Wu et al., 2016). Thus, identifying potential therapeutic targets and developing new drugs for NAFLD would have substantial clinical value.

The development of NAFLD is a complex multi-factorial process with strong genetic, environmental and metabolic contributions. The traditional "two-hit" hypothesis, which is widely accepted as a framework for guiding researches in the area, states that insulin resistance and increased FFA contribute to excessive lipid accumulation in hepatocytes in the first hit, while the second hit represents increased oxidative stress initiating lipid peroxidation and inflammatory insult to the liver (Basaranoglu et al., 2013). Recently, the "multiple-hit" hypothesis has been proposed as the more logical mechanism (Buzzetti et al., 2016).

Although comprehensive studies have been carried out to investigate the complicated molecular mechanism of NAFLD, its pathogenesis and progression still remain elusive. Proteomics is a powerful tool for the identification of novel biomarkers and potential therapeutic targets for NAFLD. iTRAQ has been widely applied in comparative proteomics due to the advantages of high throughput, high sensitivity and superior accuracy over conventional approaches (Unwin et al., 2010; Lim et al., 2014). Currently, increasing efforts have been made to identify active ingredients of herbal extracts, which have minimal side effects and aim at multiple targets, to expand the treatment options for NAFLD (Hsu et al., 2016).

Oxymatrine (OMT), a potent monosomic alkaloid derived from the root of Sophora flavescens Ait, has been reported to possess anti-inflammatory, anti-oxidative and hepatoprotective activities (Lu et al., 2016; Zhao et al., 2016; Li et al., 2017). Available studies revealed that OMT attenuated hepatic steatosis through the down-regulation of sterol regulatory element binding transcription factor 1 (SREBF1) and up-regulation of peroxisome proliferator activated receptor alpha (PPAR $\alpha$ ) mediated metabolic pathway (Shi L.J. et al., 2013). However, these studies only superficially detected the mRNA and protein expressions of SREBF1 and PPAR $\alpha$. The underlying mechanism of OMT on improving steatosis is incompletely understood.

Therefore, in the present study, a rat model of hepatic steatosis was created by high fat and high sucrose diet (HFHSD) feeding and treated with OMT. The aim of our study was to identify a list of DEPs in the liver by iTRAQ-based proteomic analysis to unveil the potential therapeutic targets of OMT for improving steatosis.

\section{MATERIALS AND METHODS}

\section{Reagents and Equipments}

Oxymatrine (purity > 98\%) was purchased from Sigma-Aldrich. Hepatic TG and FFA assay kits were from Nanjing Jiancheng Bioengineering Institute. Sequencing-grade trypsin was from Promega. iTRAQ reagent-8 plex multiplex kit was obtained from Applied Biosystems. Triple TOF 5600 mass spectrometer and Eksigent nanoLC-1D plus liquid chromatography were from SCIEX. Rabbit anti-Plin2, rabbit anti-L-FABP, rabbit anti-FASN, rabbit anti-Sirt1 and mouse anti-SCD1 monoclonal antibodies were from Abcam. Rabbit anti-AMPK $\alpha$ (phospho-Thr172) and rabbit anti-AMPK $\alpha$ monoclonal antibodies were from Cell Signaling Technology.

\section{Animals, Ethics Statement and Treatment Protocol}

Male Sprague-Dawley rats were obtained from Sino-British SIPPR/BK Lab Animal Ltd (SCXK [Shanghai] 2013-0016) and reared in the specific pathogen free facility of the Experimental Animal Research Center, Zhejiang Chinese Medical University. All animals received humane care with strict accordance to the criteria outlined in the Guide for the Care and Use of Laboratory Animals. The study was reviewed and approved by the Committee on Animal Research and Ethics of Zhejiang Chinese Medical University (number ZSLL-2014-36).

Steatosis was induced by HFHSD feeding for 8 weeks. Thirty rats weighing 180-200 g were equally randomized into three groups: control group $(n=10)$, model group $(n=10)$ and OMT-treated group $(n=10)$. The rats in the model group and OMT-treated group were fed with HFHSD, which consist of $74.25 \%$ standard chow, $10 \%$ sucrose, $0.5 \%$ cholesterol, $5 \%$ egg yolk powder, $10 \%$ lard, $0.25 \%$ sodium cholate. The rats in OMTtreated group additionally received intragastric administration of OMT at a dose of $100 \mathrm{mg} / \mathrm{kg} /$ day. The control rats were fed a standard chow and received normal saline intragastrically.

\section{Serum Biochemical Assays}

The activities of serum ALT and AST and the levels of triglyceride (TG) and total cholesterol (TC) were determined by an Beckman Coulter AU5800 automatic biochemical analyzer.

\section{Liver Histological Examination}

Liver specimens were fixed in $4 \%$ paraformaldehyde, dehydrated in a graded alcohol series and embedded in paraffin. Sections of $4 \mu \mathrm{m}$ thickness were stained with hematoxylin and eosin (H\&E). To determine hepatic lipid accumulation, fresh liver tissues were embedded in OCT compound and frozen in liquid nitrogen. Frozen sections $(10 \mu \mathrm{m})$ were stained with Oil Red O. The percentage of Oil Red-positive staining area was calculated by using ImagePro Plus software from five to seven views per animal.

\section{Hepatic Lipid Profiles}

The levels of TG and FFA in the liver were determined using commercial kits according to the manufacturer's protocols. 


\section{Protein Extraction, Digestion, and iTRAQ Labeling}

Frozen liver tissues were ground into powder using liquid nitrogen. The powder was homogenated in ice-cold SDS lysis buffer (containing $50 \mathrm{mM}$ Tris, $0.1 \%$ SDS, $1 \%$ Triton X-100 and protease inhibitor cocktail). The lysate was centrifuged at $12,000 \mathrm{~g}, 4^{\circ} \mathrm{C}$ for $15 \mathrm{~min}$. The supernatant was collected and precipitated with ice-cold acetone $(1: 5, \mathrm{v} / \mathrm{v})$ at $-20^{\circ} \mathrm{C}$ overnight. The pellet was collected by centrifugation, washed twice with ice-cold acetone, dried and resolved with sample buffer (7 M urea, $2 \mathrm{M}$ thiourea, $50 \mathrm{mM}$ DTT, $1 \mathrm{mM}$ PMSF, $50 \mathrm{mM}$ Tris, $1 \mathrm{mM}$ RNAse, and $1 \mathrm{mM}$ DNAse). Protein concentration was determined and equal amount of total protein from several rats in the same group were pooled together as a biological replicate to alleviate the individual variability. Two or three biological replicates were acquired.

A total of $200 \mu \mathrm{g}$ of pooled proteins from each group was reduced, alkylated, and digested by sequencing-grade trypsin. Briefly, the samples were was incubated with $200 \mu$ l reducing buffer (10 mM DTT, $8 \mathrm{M}$ urea, $100 \mathrm{mM} \mathrm{TEAB,} 150 \mathrm{mM}$ Tris-HCl, $\mathrm{pH}$ 8.0) for $1 \mathrm{~h}$ at $60^{\circ} \mathrm{C}$, cooled to room temperature, alkylated with $50 \mathrm{mM}$ iodoacetamide (IAA) for $1 \mathrm{~h}$ in darkness and subjected to $10 \mathrm{kDa}$ ultrafiltration. The sediment were collected and digested by sequencing-grade trypsin at $37^{\circ} \mathrm{C}$ for $16 \mathrm{~h}$, with ratios of protein to trypsin of 50:1, and labeled using iTRAQ reagent- 8 plex Multiplex Kit according to the manufacturer's protocol (Liu X. et al., 2017).

\section{Reversed Phase Liquid Chromatography Fractionation}

The iTRAQ-labeled peptide mixtures were separated using an Agilent Zorbax Extend RP column (C18, $5 \mu \mathrm{m}$, $150 \mathrm{~mm} \times 2.1 \mathrm{~mm})$. Mobile phases A $(2 \% \mathrm{ACN}$ in water) and B ( $98 \% \mathrm{ACN}$ in water) were used for gradient. The solvent gradient was set as described previously (Hu et al., 2018). Peptides were separated at a fluent flow rate of $300 \mu \mathrm{L} / \mathrm{min}$ and monitored at $210 \mathrm{~nm}$ and $280 \mathrm{~nm}$. Dried samples were harvested from 8 to $50 \mathrm{~min}$ and elution buffer were collected in every minute and numbered from 1 to 10 with pipeline. The separated peptides were lyophilized for MS detection.

\section{LC-MS/MS Analysis}

All analyses were performed by a Triple TOF 5600 mass spectrometer equipped with a Nanospray III source. Samples were separated by a reverse-phase C18 column $(15 \mathrm{~cm} \times 75 \mu \mathrm{m}$, $3 \mu \mathrm{m}, 120 \AA$ ) on an Eksigent nanoLC-1D plus system. Mobile phase $A=2 \% \mathrm{ACN} / 0.1 \% \mathrm{FA}$ and $B=95 \% \mathrm{ACN} / 0.1 \% \mathrm{FA}$. The flow rate was $300 \mathrm{~nL} / \mathrm{min}$ and linear gradient was set as described previously (Hu et al., 2018).

Data were acquired with a $2.4 \mathrm{kV}$ ion spray voltage, 35 psi curtain gas, 5 psi nebulizer gas, and an interface heater temperature of $150^{\circ} \mathrm{C}$. The MS scanned between 400 and 1500 with an accumulation time of $250 \mathrm{~ms}$. For IDA, $30 \mathrm{MS} / \mathrm{MS}$ spectra ( $80 \mathrm{~ms}$ each, mass range 100-1500) of MS peaks above intensity 260 and having a charge state of between 2 and 5 were acquired. A rolling collision energy voltage was used for CID fragmentation for MS/MS spectra acquisitions. Mass was dynamically excluded for $22 \mathrm{~s}$ (Yu et al., 2019).

\section{Database Search}

The original MS/MS file data were analyzed by ProteinPilot Software v5.0. Processing parameters were set as follows: iTRAQ 8-plex quantification, cysteine modified with IAA; biological modifications were selected as ID focus, trypsin digestion; protein quantification and normalization were checked by the Background Correction, Quantitate and Bias Correction (Yao et al., 2018). Proteins with at least $95 \%$ confidence determined by Protein Pilot Unused scores $(\geq 1.3)$ were reported, and the FDR was set up less than $1 \%$. Fold changes $\geq 1.3$ were considered significant.

\section{Bioinformatics Analysis}

The interaction networks of DEPs were analyzed by STRING database ${ }^{1}$. The BP, CC, and MF were analyzed by GO database ${ }^{2}$. We defined the significance of GO enrichment according to a $P$ value $<0.05$. The pathway analysis was performed by KEGG database ${ }^{3}$.

\section{Western Blot Analysis}

Samples were prepared as described previously (Xu et al., 2016). Equal amounts $(30-50 \mu \mathrm{g})$ of protein were loaded on $8 \%$ or $12 \%$ SDS-polyacrylamide gel, separated and transferred to polyvinylidene difluoride membranes. The blots were probed with rabbit monoclonal antibodies against Plin2 (1:1000), L-FABP (1:1000), FASN (1:1000), Sirt1 (1:1000), AMPK $\alpha$ (1:1000), AMPK $\alpha$ (Thr172) (1:1000) and mouse monoclonal antibody against SCD1 (1:1000) followed by HRP-conjugated goat anti-rabbit IgG or rabbit anti-mouse IgG. Signals were visualized by enhanced chemiluminescence detection.

\section{Statistical Analysis}

Statistical Package for the Social Sciences (SPSS version 20.0) software was used for the statistical analysis. Data are presented as mean \pm standard deviation (SD) and analyzed by one-way ANOVA. Bonferroni's correction was performed to adjust for multiple comparisons. $P<0.05$ was considered as statistically significant.

\section{RESULTS}

\section{OMT Reduces Body Weight and Liver Weight in Steatosis Rats}

Prior to the experiment, no differences were observed in body weight among the three groups. After 8 weeks of HFHSD feeding, macroscopic appearances of the livers from the model rats were enlarged, yellowish and greasy, whereas the control rats displayed brown, smooth, and shiny liver tissues. Accordingly, the animals

\footnotetext{
${ }^{1}$ http://string-db.org

${ }^{2} \mathrm{http}$ //geneontology.org/

${ }^{3}$ http://www.genome.jp/kegg/mapper.html
} 
in the model group showed significantly increased body weight and liver weight compared to those in the control. The abnormal macroscopic appearance of the liver was significantly improved by OMT treatment. Furthermore, OMT effectively decreased the body weight as well as the liver weight (Figures 1A-C).

\section{OMT Alleviates Hepatic Fatty Degeneration}

Representative images of randomly selected sections by H\&E and Oil Red $\mathrm{O}$ staining were shown in Figure 1D. The control rats exhibited a normal liver architecture with well-arranged hepatic

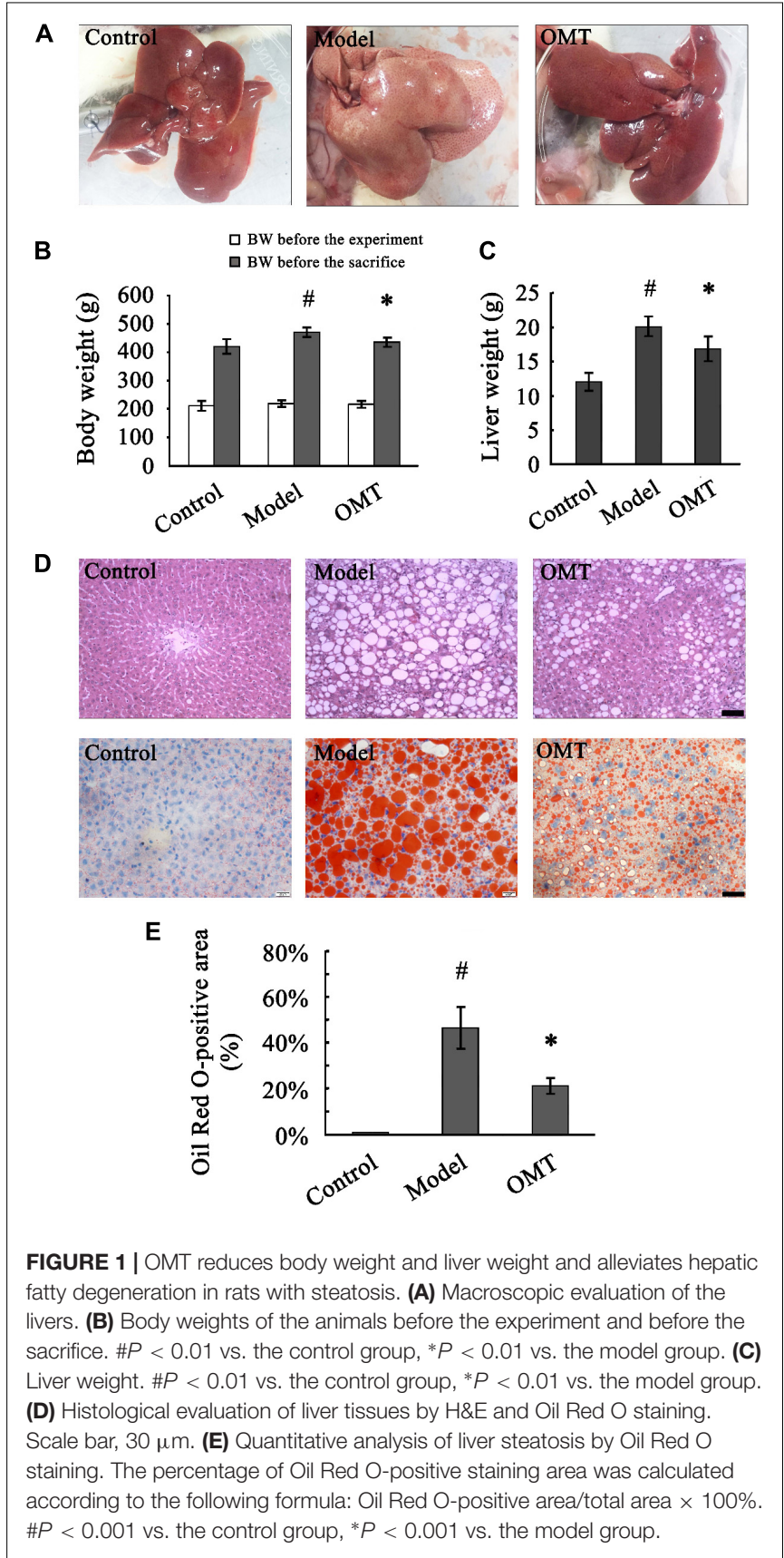

lobules. Severe fatty degeneration with destroyed structure of normal hepatic lobules was observed in the livers of the model rats. The percentage of Oil Red O-positive staining area in the model group was significantly higher than that in the control $(46.50 \pm 9.08 \%$ vs. $0.90 \pm 0.13 \%)$. The fatty degeneration was noticeably improved by OMT treatment with the percentage of Oil Red O-positive staining area $(21.15 \pm 3.40 \%)$ significantly decreased compared to the model group (Figure 1E).

\section{OMT Improves Serum and Hepatic Lipid Profiles}

Slightly but not statistically increased serum ALT and AST levels were detected in the model group compared to the control and OMT-treated groups (Figures 2A,B). Compared to the control group, the levels of serum TG and TC in the model group were remarkably increased $(2.68 \pm 0.35 \mathrm{mmol} / \mathrm{L}$ vs. $1.22 \pm 0.12 \mathrm{mmol} / \mathrm{L}$ and $2.67 \pm 0.29 \mathrm{mmol} / \mathrm{L}$ vs. $1.44 \pm 0.12 \mathrm{mmol} / \mathrm{L}$, respectively). OMT treatment significantly reduced serum TG and TC levels to $2.13 \pm 0.32 \mathrm{mmol} / \mathrm{L}$ and $2.15 \pm 0.33 \mathrm{mmol} / \mathrm{L}$,
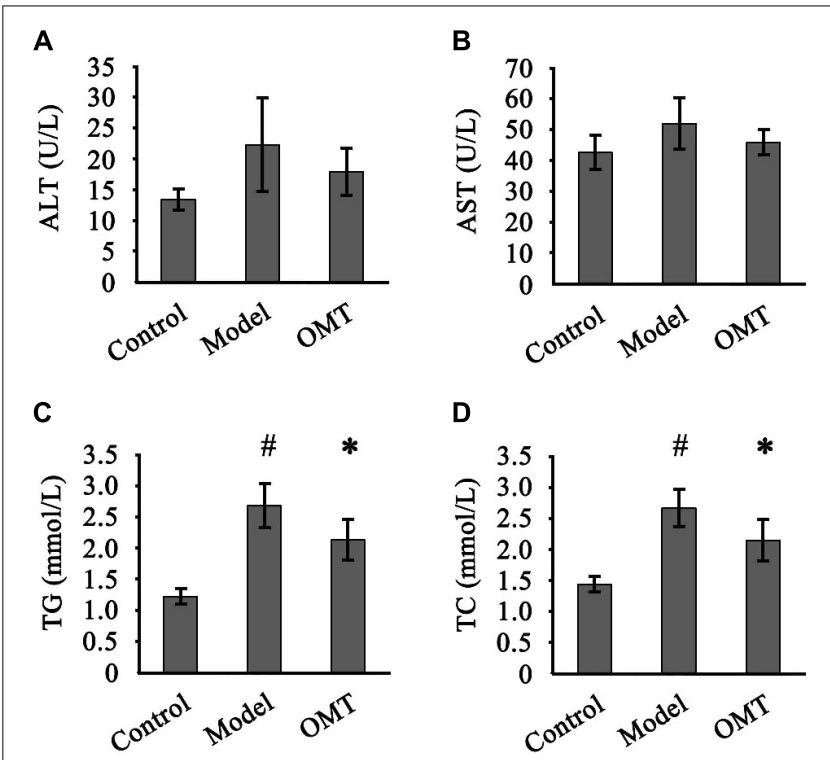

D
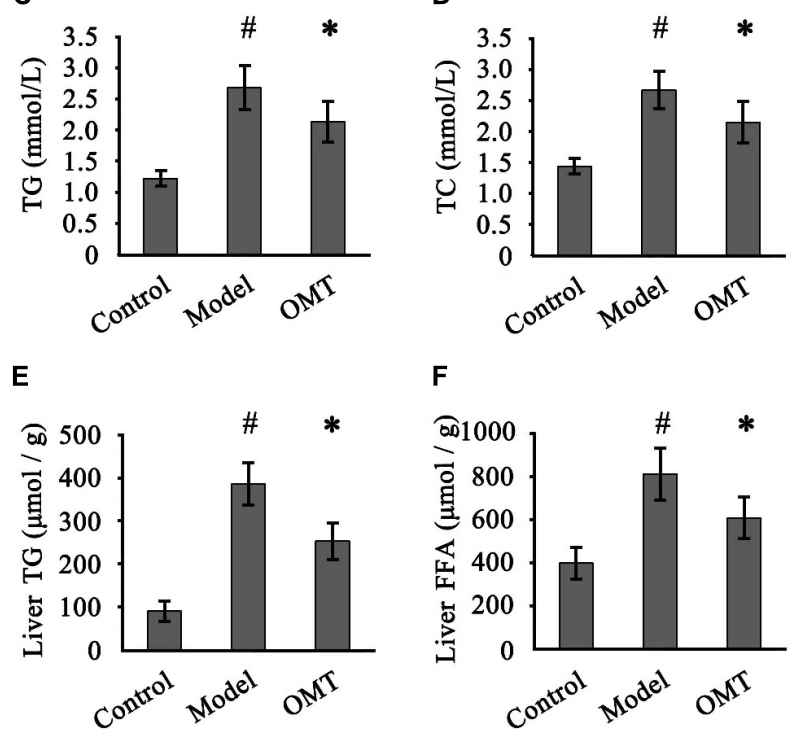

$\mathbf{F}$

FIGURE 2 | OMT improves serum and hepatic lipid profiles. Serum levels of ALT (A) and AST (B) among the three groups were detected. Serum levels of TG (C) and TC (D). Hepatic levels of TG (E) and FFA (F). Data are representative of 10 rats in each group and expressed as mean $\pm \mathrm{SD}$. $\# P<0.01$ vs. the control group, ${ }^{*} P<0.01$ vs. the model group. 
respectively (Figures 2C,D). Similarly, the model group displayed significantly higher hepatic TG and FFA levels than the control $(386.2 \pm 47.8 \mu \mathrm{mol} / \mathrm{g}$ vs. $90.8 \pm 22.7 \mu \mathrm{mol} / \mathrm{g}$ and $812.0 \pm 118.3 \mu \mathrm{mol} / \mathrm{g}$ vs. $398.4 \pm 72.6 \mu \mathrm{mol} / \mathrm{g}$, respectively). OMT significantly decreased hepatic TG and FFA levels (Figures 2E,F).

\section{iTRAQ Quantitative of DEPs}

iTRAQ-based proteomic analysis was used to detect DEPs in each group. After merging the data from two or three independent biological replicates, a total of 2859 proteins were identified and quantified against the Rat Database (FDR $<1 \%$ and containing at least two unique peptides). 173 DEPs $(p<0.05$ and changes $>1.3$-fold) were identified between the model group and the control group, of which 77 proteins were upregulated and 96 proteins were down-regulated. 173 DEPs were identified between the OMT-treated group and the model group, of which 88 proteins were up-regulated and 85 proteins were down-regulated. Furthermore, 301 differential proteins were identified between the OMT-treated group and the control group with 141 proteins up-regulated and 60 proteins downregulated (Figure 3 ).

We classified all DEPs into two categories: steatosis-specific DEPs, which were up-regulated or down-regulated significantly in the liver of the model group compared to the control, and OMT-related DEPs. OMT-related DEPs include a bunch of steatosis-specific DEPs, which were regulated in an opposite

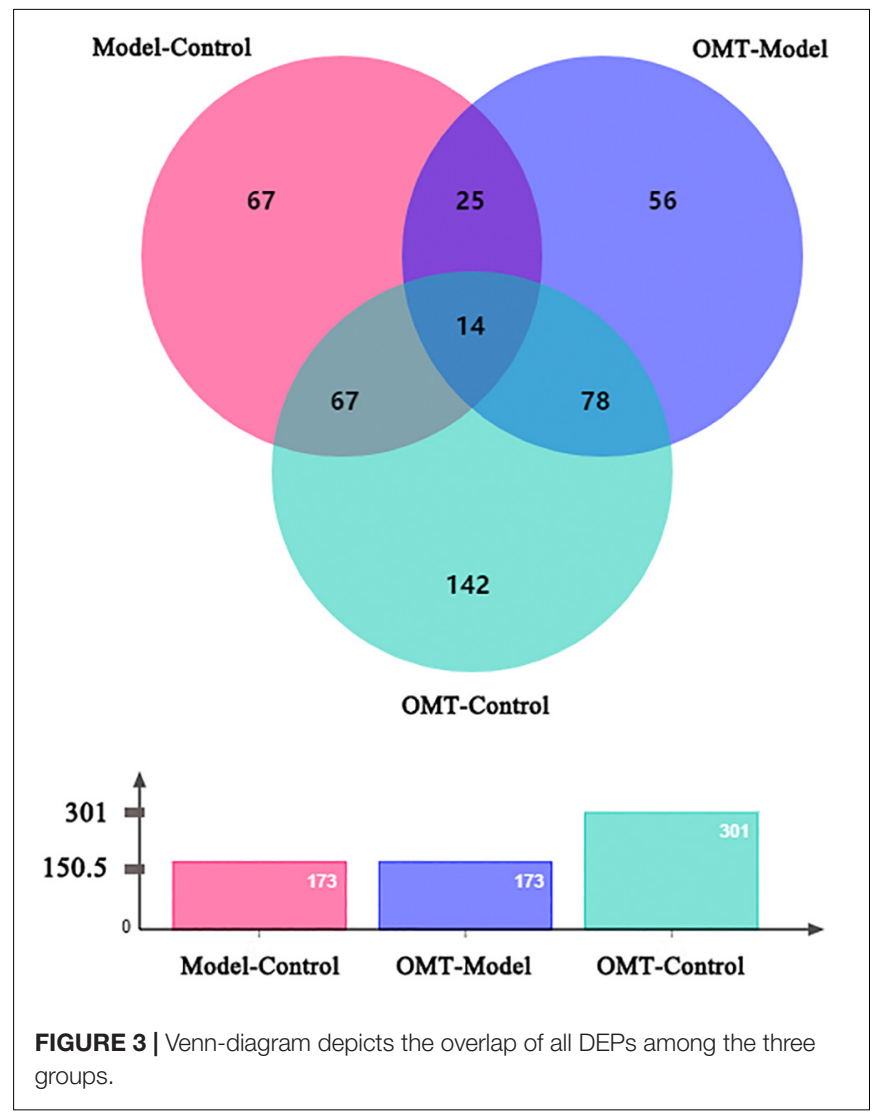

way following OMT treatment. Representative proteins included perilipin 2 (Plin2), liver fatty acid-binding protein (L-FABP, also known as FABP1), FASN and stearoyl -CoA desaturase 1 (SCD1). The expressions of some proteins remained unchanged throughout HFHSD feeding, but significantly changed by the administration of OMT. After the removal of the proteins overlapped with steatosis-specific DEPs, there were 177 DEPs between the OMT-treated group and control group. These proteins were also defined as OMT-related DEPs.

Representative OMT-related DEPs were shown in Table 1. These proteins were mainly involved in fatty acid metabolism (Fasn, Scd1, Fads2, Fdps, Plin2), fatty acid degradation (Ehhadh, Acs11), PPAR signaling pathway (Fads2, Ehhadh, Acsl1, Fabp1), non-alcoholic fatty liver disease (Ehhadh, Pklr) and AMPK signaling pathway (Fasn, Scd1, Hnf4a).

\section{Functional Classifications of DEPs}

All these DEPs were analyzed by searching GO database to determine their participation in BP, CC and MF. As shown in Figure 4, the DEPs were mainly located in the cytoplasm, intracellular membrane-bounded organelle, membrane and nucleus. Their major MFs were catalytic activity, ion binding, protein binding, organic cyclic compound binding and heterocyclic compound binding. OMT-related DEPs were mainly involved in processes of small molecule metabolism, organic substance biosynthesis, organic cyclic compound metabolism, response to organic substance, organic acid metabolism, transport and oxidation-reduction.

TABLE 1 | Representative OMT-related DEPs detected by iTRAQ analysis.

\begin{tabular}{|c|c|c|c|c|c|}
\hline $\begin{array}{l}\text { Protein } \\
\text { name }\end{array}$ & $\begin{array}{l}\text { Uniprot } \\
\text { accession }\end{array}$ & $\begin{array}{l}\text { Model/Control } \\
\text { (fold) }\end{array}$ & $P$ value & $\begin{array}{l}\text { OMT/Model } \\
\text { (fold) }\end{array}$ & $P$ value \\
\hline Plin2 & $\begin{array}{l}\operatorname{tr|} \text { Q5U2U5| } \\
\text { Q5U2U5_RAT }\end{array}$ & 2.80 & 2.044E-05 & 0.54 & 0.000 \\
\hline Fabp1 & $\begin{array}{l}\text { sp| P02692| } \\
\text { FABP1_RAT }\end{array}$ & 2.56 & 0.001 & 0.52 & 0.003 \\
\hline Fasn & $\begin{array}{l}\text { sp| P12785| } \\
\text { FAS_RAT }\end{array}$ & 3.12 & 0.000 & 0.46 & 0.004 \\
\hline Scd1 & $\begin{array}{l}\text { sp| P07308| } \\
\text { ACOD1_RAT }\end{array}$ & 2.43 & 0.000 & 0.48 & 0.000 \\
\hline Fads2 & $\begin{array}{l}\text { sp| Q9Z122| } \\
\text { FADS2_RAT }\end{array}$ & 0.53 & 0.017 & 1.98 & 0.003 \\
\hline $\mathrm{Hnf} 4 \mathrm{a}$ & $\begin{array}{l}\text { tr| G3V750| } \\
\text { G3V750_RAT }\end{array}$ & 1.89 & 0.015 & 0.49 & 0.005 \\
\hline Pklr & $\begin{array}{l}\text { sp| P12928| } \\
\text { KPYR_RAT }\end{array}$ & 2.23 & 0.007 & 0.54 & 0.006 \\
\hline Fdps & $\begin{array}{l}\text { tr| F1LND7| } \\
\text { F1LND7_RAT }\end{array}$ & 0.35 & 0.000 & 1.97 & 0.001 \\
\hline Rab18 & $\begin{array}{l}\text { sp| Q5EB77| } \\
\text { RAB18_RAT }\end{array}$ & 2.16 & 0.002 & 0.58 & 0.001 \\
\hline Ehhadh & $\begin{array}{l}\text { sp| P07896| } \\
\text { ECHP_RAT }\end{array}$ & 0.56 & 0.005 & 1.76 & 0.001 \\
\hline Acsl1 & $\begin{array}{l}\text { sp| P18163| } \\
\text { ACSL1_RAT }\end{array}$ & 1.88 & 0.001 & 0.62 & 0.004 \\
\hline Ppox & $\begin{array}{l}\text { tr| D3ZVN7| } \\
\text { D3ZVN7_RAT }\end{array}$ & 2.03 & 0.007 & 0.60 & 0.003 \\
\hline
\end{tabular}




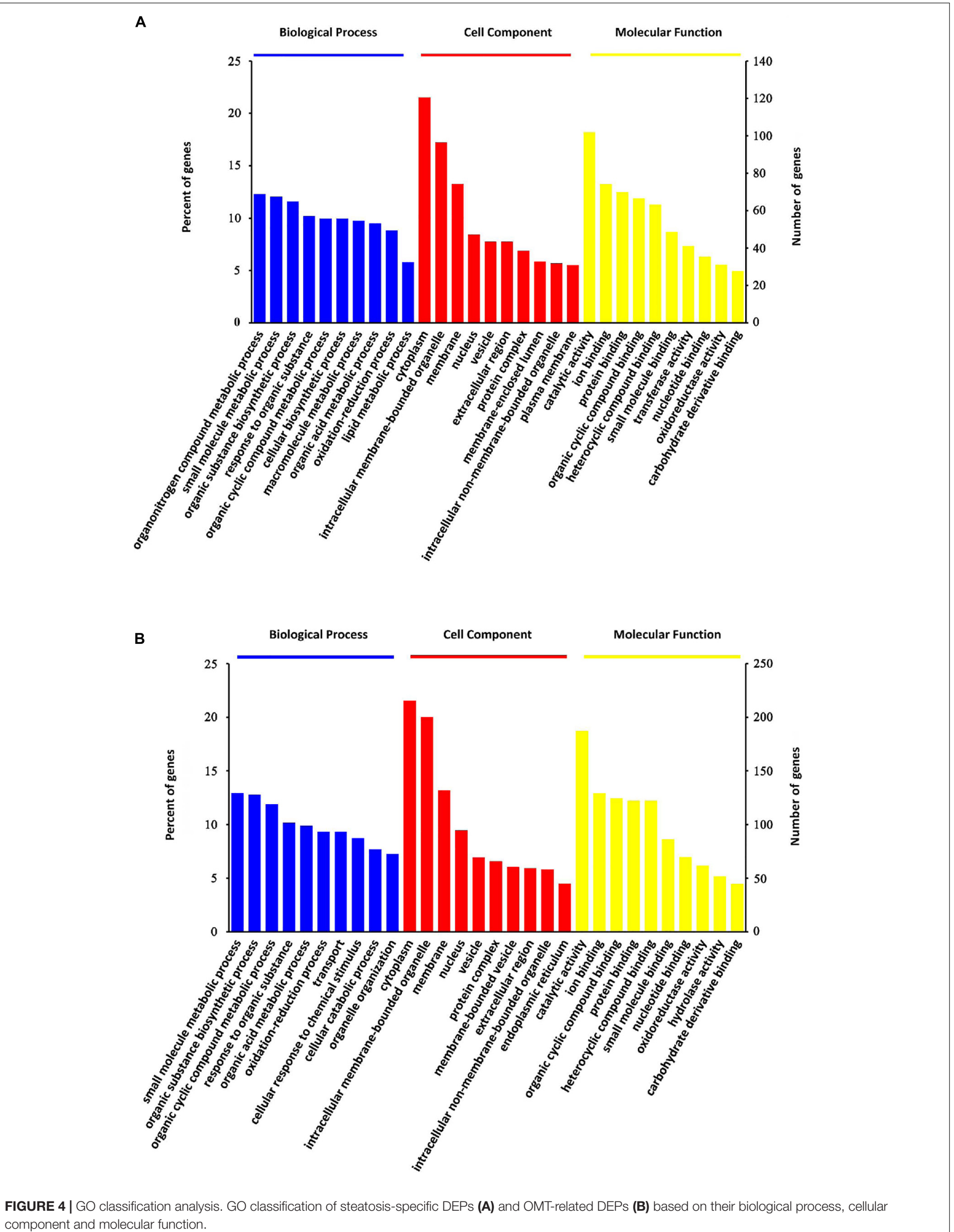




\section{Pathway Enrichment and Protein-Protein Network Analysis}

KEGG enrichment was performed to uncover the signal transduction pathways that DEPs may participate in. Steatosisspecific DEPs were mainly involved in carbon metabolism, biosynthesis of amino acids, arginine biosynthesis, cysteine and methionine metabolism, PPAR signaling pathway, glycine, serine and threonine metabolism, alanine, aspartate and glutamate metabolism, metabolism of xenobiotics by cytochrome P450, fatty acid metabolism and metabolic pathways. The top 10 signaling pathways that OMT-related DEPs participate in included tyrosine metabolism, steroid hormone biosynthesis, starch and sucrose metabolism, retinol metabolism, PPAR signaling pathway, peroxisome, glutathione metabolism, drug metabolism-cytochrome $\mathrm{P} 450$, fatty acid degradation and metabolic pathways (Figure 5). As shown in Figure 6, String network analysis identified various possible direct and indirect interactions among these DEPs. These proteins were mainly involved in fatty acid metabolism, fatty acid degradation, PPAR signaling pathway, peroxisome, non-alcoholic fatty liver disease, arginine biosynthesis, AMPK signaling pathway, biosynthesis of unsaturated fatty acids and biosynthesis of amino acids.

\section{Validation of Potential Therapeutic Targets by Western Blot Analysis}

Four significantly changed proteins: L-FABP, Plin2, FASN, and SCD1 were chosen for validation by western blot analysis. As shown in Figure 7, the expressions of L-FABP, Plin2, FASN, and SCD1 in the liver were significantly increased in the model group compared to the control and were 3.25-fold, 2.48-fold, 3.34 -fold and 2.56-fold over the control, respectively. OMT treatment caused significant reductions in the levels of L-FABP, Plin2, FASN, and SCD1 by $52.92,55.63,52.69$, and $57.42 \%$, respectively. These results are concordant with our findings from iTRAQ analysis.

\section{OMT Increases Sirt1 Expression and AMPK $\alpha$ Phosphorylation}

As shown in Figure 8, the expression of Sirt1 in the liver of the model rats was significantly reduced to $58.03 \%$ of the control. OMT caused a significant increase in Sirt1 expression up to 1.89fold over the model group. No significant changes were detected in the protein expression of AMPK $\alpha$ among the three groups. However, Thr172 phosphorylation of AMPK $\alpha$ was significantly decreased compared to the control. In OMT-treated group, Thr172 phosphorylation of AMPK $\alpha$ was significantly increased and was 2.63 -fold over the model group.

\section{DISCUSSION}

Current innovative strategies to treat NAFLD include identifying active ingredients of herbal extracts that can suppress lipid accumulation in the liver (Liu Q. et al., 2017; Zhang et al., 2018). The present study is not the first one reporting the anti-steatotic action of OMT. But importantly, this is for the first time displaying hepatic proteomic response to oxymatrine treatment in steatosis rats by iTRAQ analysis. Furthermore, our data have provided the novel evidence that OMT activates hepatic Sirt1/AMPK signaling which might be the potential therapeutic target of OMT for improving steatosis.

Although the precise mechanisms underlying the pathogenesis and progression of NAFLD still remain unclear, excessive lipid accumulation in the liver serves as a precursor for steatosis. It could stem from increased uptake of FFAs, elevated de novo lipogenesis and impaired fatty acid $\beta$ oxidation. SREBP is a transcription activating factor of almost all genes that control the synthesis of fatty acids and TG in the liver. PPAR $\alpha$ is another key regulator of the complex regulatory network of hepatic lipid metabolism (Cobbina and Akhlaghi, 2017). Therapeutic options targeting the improvement of lipid metabolism in the liver are crucial for the management of NAFLD. By creating HFHSD-induced steatosis rat model, we have provided strong support for OMT as a potential candidate for NAFLD treatment with high therapeutic efficiency. The body weight and liver weight of animals with steatosis were significantly reduced by OMT treatment. The sick greasy macroscopic appearances of the livers were remarkably improved. The serum levels of TG and TC as well as the hepatic TG and FFA levels were significantly decreased. Histopathological examination demonstrated that OMT effectively alleviated fatty degeneration in the liver. Moreover, OMT significantly attenuated lipid droplets (LDs) formation in oleic acid-induced steatotic hepatocytes and reduced intracellular TG and TC levels (see Supplementary Material).

Prior to the present study, limited literatures have documented the protective effect of OMT against hepatic steatosis (Shi L. et al., 2013). However, the starting point of these researches was based on the important roles of SREBF1 and PPAR $\alpha$ in lipid synthesis and fatty acid $\beta$-oxidation, respectively. There were not any more details except the determination of the mRNA and protein expressions of SREBF1 and PPAR $\alpha$. A clear and detailed mechanism by which OMT improves steatosis remains largely unknown. In this study, by using iTRAQ-based proteomic method, a bunch of DEPs which up-regulate or down-regulate significantly by OMT treatment were identified. The data of GO analysis suggested that OMT-related DEPs were mainly located in the cytoplasm, intracellular membrane-bounded organelle, membrane and nucleus, participating mainly in the processes of small molecule metabolism, organic substance biosynthesis, organic cyclic compound metabolism, response to organic substance, organic acid metabolism, transport and oxidationreduction. KEGG enrichment revealed that they were closely associated with following pathways: tyrosine metabolism, steroid hormone biosynthesis, starch and sucrose metabolism, retinol metabolism, PPAR signaling pathway, peroxisome, glutathione metabolism, drug metabolism-cytochrome P450, fatty acid degradation and metabolic pathways. Plin2, L-FABP, FASN, and SCD1 were selected as the most significantly differentially expressed targets.

Plin2 is constitutively located on the surface of LDs. It has been considered a reliable and sensitive marker for LDs and correlates positively with cytosolic TG content in hepatocytes. 
A

Ubiquinone and other terpenoid-quinone biosynthesis -

PPAR signaling pathway -

Porphyrin and chlorophyll metabolism -

Pentose and glucuronate interconversions -

Non-alcoholic fatty liver disease (NAFLD) -

Metabolism of xenobiotics by cytochrome P450 -

Metabolic pathways -

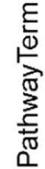

Glycine, serine and threonine metabolism -

Glutathione metabolism -

Fatty acid metabolism -

Drug metabolism - cytochrome P450 -

Cysteine and methionine metabolism -

Carbon metabolism -

Biosynthesis of unsaturated fatty acids -

Biosynthesis of amino acids -

Arginine biosynthesis -

Alanine, aspartate and glutamate metabolism -

2-Oxocarboxylic acid metabolism -

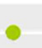

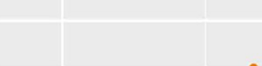

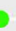

Number

- 10

- 20

- 30

D 40

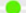

P_value

0.0075

0.0050

0.0025

-

0

0.1

RichFactor

B

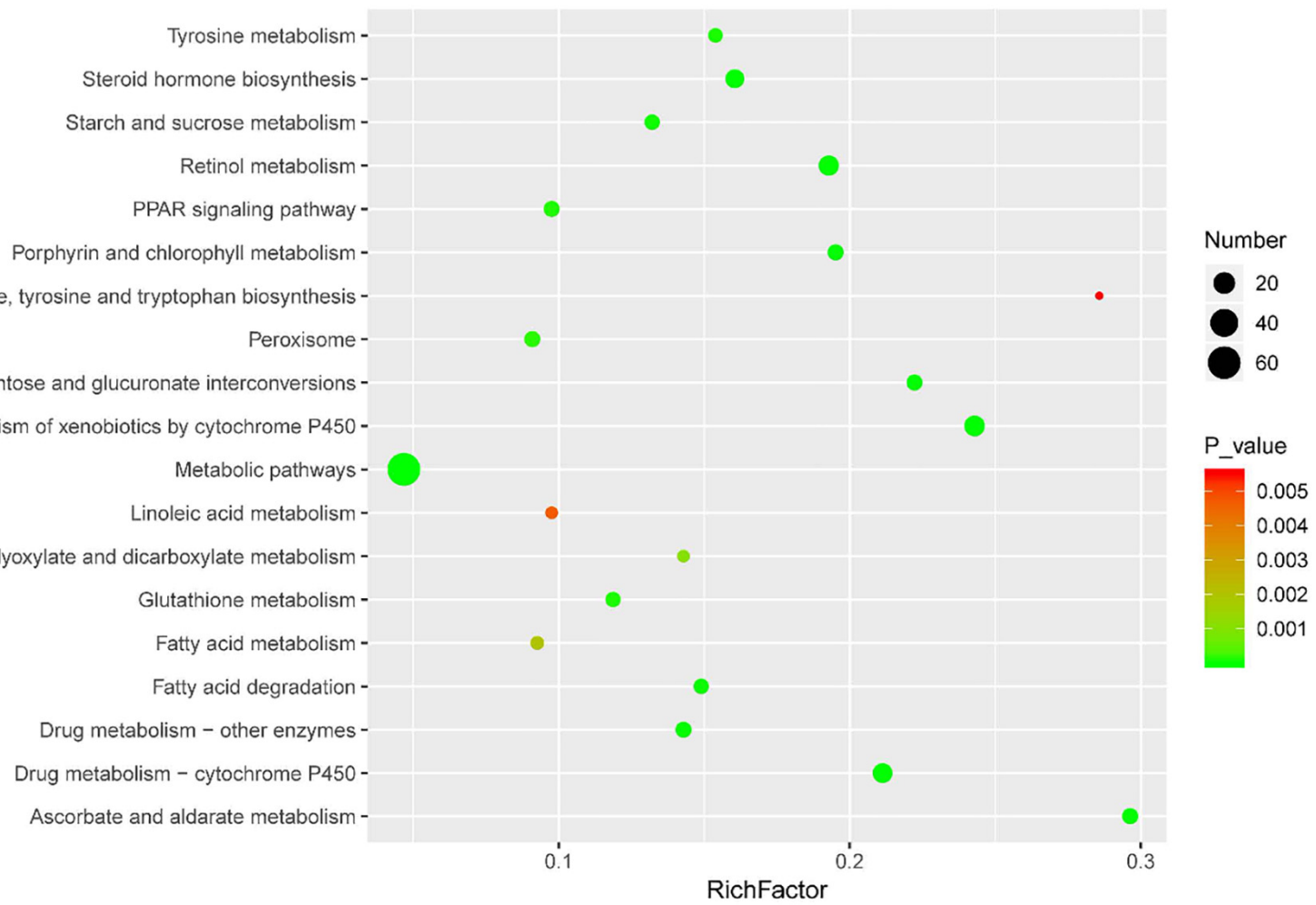

FIGURE 5 | Scatter diagram of enriched KEGG pathways. Enriched KEGG pathways of steatosis-specific DEPs (A) and OMT-related DEPs (B). Degree of enrichment was determined by the number of genes that enriched in one pathway, Rich factor and $P$ value and. $Y$-axis represents the name of signaling pathway, $X$-axis represents the Rich factor. Point size means the number of differential expression genes in specific pathway, and the color of point means the range of $P$ value. 


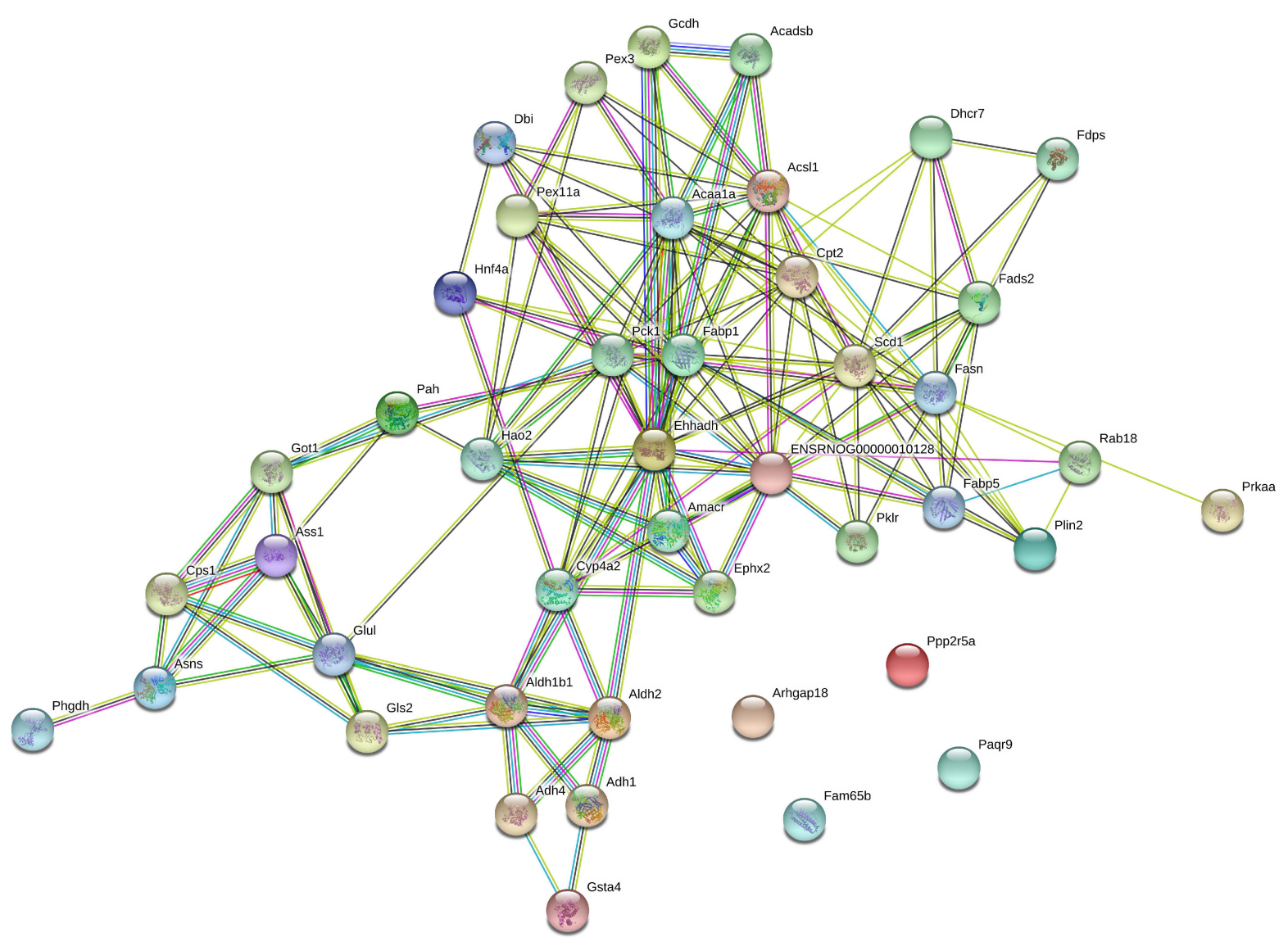

FIGURE 6 | String network analysis of DEPs identified by iTRAQ-LC-MS/MS.

Plin2 plays a key role in fatty acid uptake, stabilization of LDs, lipid transport and storage. Plin2 regulated lipid exchange from LDs by facilitating direct protein-lipid interactions on the LDs surface (McIntosh et al., 2012). Study of clinical liver biopsy discovered that Plin2 mainly targeted inflamed ballooned hepatocytes. The frequency of Plin2-positive ballooned hepatocytes was correlated to inflammation and NAFLD activity score (Fujii et al., 2009). Suppression of Plin2 expression by global or liver-specific ablation of plin2 gene resulted in decreased hepatic lipid accumulation and protected against diet-induced liver steatosis, inflammation and fibrosis (McManaman et al., 2013; Najt et al., 2016). Furthermore, whole-body loss of Plin2 exerts a protective effect in animals exposed long-term Western diet in part by suppressing hepatic SRBEP-1 and SRBEP-2 activity (Libby et al., 2016).

L-FABP is a small $14 \mathrm{kDa}$ soluble protein and abundantly expressed in hepatocytes in high concentration, accounting for $2-5 \%$ of all soluble cytosolic proteins. L-FABP functions as a transporter of fatty acid in the cytoplasm. It mediates the transport of long-chain fatty acids (LCFAs) and other lipid ligands from cytoplasm to various organelles, such as nucleus, LDs, mitochondria, peroxisome and endoplasmic reticulum (Wang et al., 2015). Ablation of L-FABP gene impaired the ability of the liver to efficiently import and transfer fatty acids into glycerolipid biosynthesis resulting in a reduction of hepatic TG accumulation, and protected against diet-induced obesity and hepatic steatosis (Newberry et al., 2006; Martin et al., 2009, 2017; Mukai et al., 2017). Studies discovered that L-FABP and PPAR $\alpha$ colocalized in the nucleus. L-FABP may serve to shuttle LCFAs into the nucleus for donating the ligands to PPAR $\alpha$ and interacts directly with PPAR $\alpha$ to influence transcriptional activity (Wolfrum et al., 2001; Huang et al., 2004). FASN and SCD1 are important lipogenesis-associated enzymes. FASN catalyzes the last step in de novo fatty acid synthesis. SREBP1c is the major transcriptional factor that regulates the expressions of FASN and SCD1 (Angeles and Hudkins, 2016; Zhang et al., 2017). FASN also controls the activation of PPAR $\alpha$ under nutrientdeficient conditions to promote the adaptive response to fasting (Jensen-Urstad and Semenkovich, 2012).

Considering that OMT-related DEPs display a wide range of functions including fatty acid uptake, synthesis, transport, storage and degradation and participating PPAR signaling pathway, we speculate that the therapeutic target of OMT may locate on the upstream of the network of lipid metabolism regulation. Available studies have revealed that sirtuin 1 (Sirt1)/adenosine monophosphate-activated protein kinase (AMPK) signaling plays a pivotal role in lipid metabolism in the liver. AMPK, which serves as a sensor of cellular energy status, is a heterotrimeric 

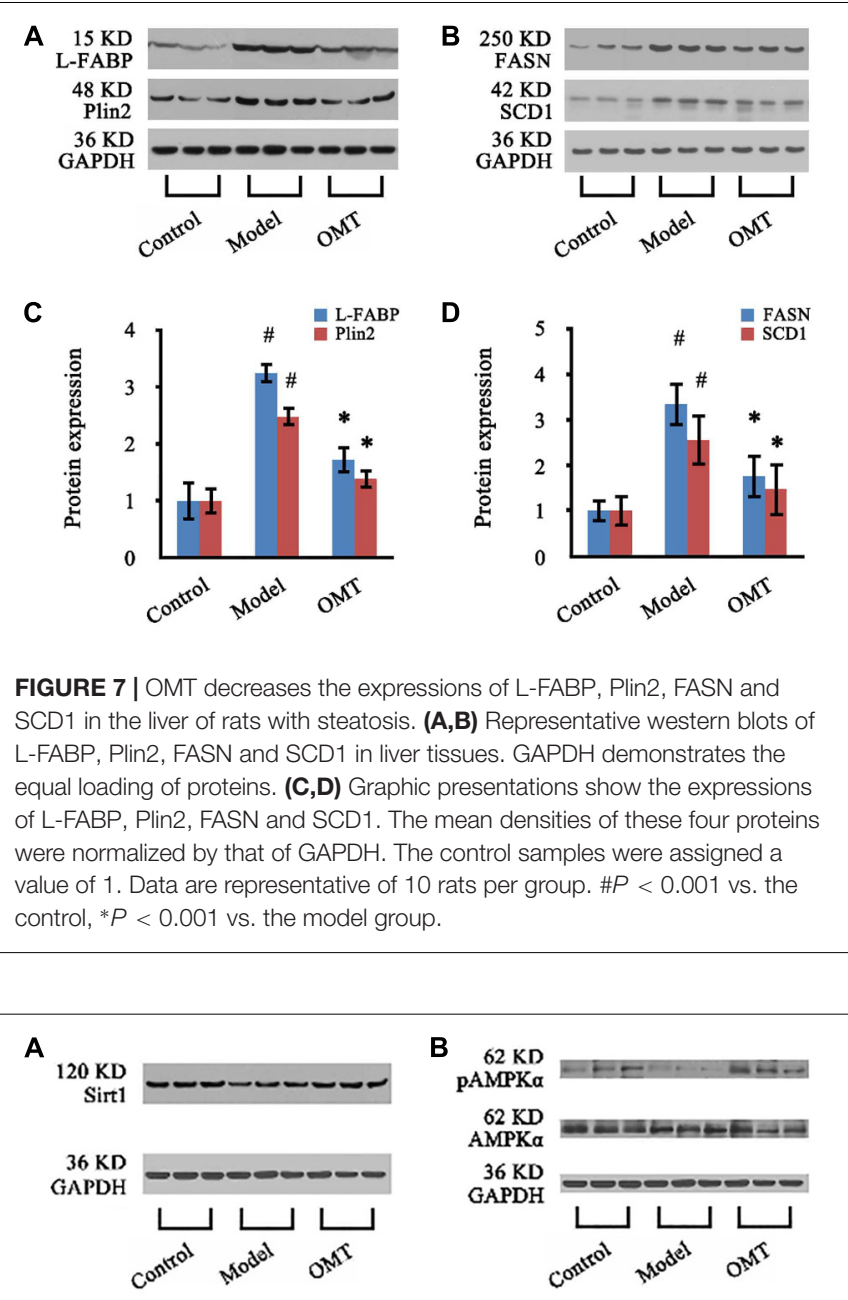

C

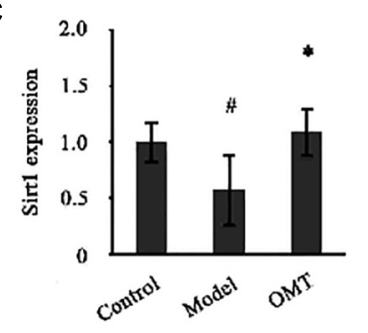

D

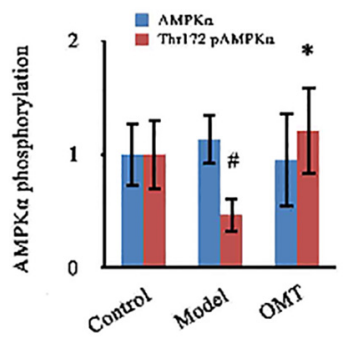

FIGURE 8 | OMT increases Sirt1 expression and AMPKa phosphorylation. (A,B) Representative western blots of Sirt1, AMPK $\alpha$ and phospho-Thr172 AMPK $\alpha$ in liver tissues. GAPDH demonstrates the equal loading of proteins. (C,D) Graphic presentations show the expressions of Sirt1 and AMPK $\alpha$ and phosphorylation of Thr172 AMPK $\alpha$. The mean densities of Sirt1 and AMPKa were normalized by that of GAPDH. AMPK $\alpha$ phosphorylation is represented as the relative ratio of the density of phospho-Thr172 AMPK $\alpha$ against that of total AMPK $\alpha$. The control samples were assigned a value of 1 . Data are representative of 10 rats per group. $\# P<0.001$ vs. the control, ${ }^{*} P<0.001$ vs. the model group.

enzyme that is composed by $\mathrm{AMPK} \alpha$ (a catalytic subunit), AMPK $\beta$ (a scaffolding subunit) and AMPK $\gamma$ (a regulatory subunit) (Day et al., 2017). AMPK activation by phosphorylation of Thr172 in a subunit decreases SREBP-1c expression to suppress lipid biosynthesis and activates PPAR $\alpha$ to promote fatty acid $\beta$-oxidation (Smith et al., 2016). Sirt1, an NAD + -dependent protein deacetylase, is a critical regulator of AMPK activity in controlling hepatocellular lipid metabolism (Liou et al., 2018). Sirt1 deacetylated and inhibited the activity of SREBP-1c (Ponugoti et al., 2010). Sirt1-deficient mice lacked AMPK activity and had increased SREBP-1c expression that triggered hepatic steatosis and obesity (Zhang et al., 2016). Hepatocyte-specific loss of Sirt1 was shown to cause PPAR $\alpha$ signal failure and a decrease in fatty acid $\beta$-oxidation (Purushotham et al., 2009). Our data indicated that OMT significantly increased hepatic expression of Sirt1, which is down-regulated in the liver of steatosis rats. Although the expression of total AMPK $\alpha$ remained unchanged, Thr172 phosphorylation of AMPK $\alpha$ was significantly increased following OMT treatment. The data have been further verified by in vitro experiment (see Supplementary Material). Thus, OMT may activate Sirt1/AMPK signaling to control the downstream key regulators of lipid synthesis, transport and degradation.

In summary, the present study has provided the evidence to confirm the efficacy of OMT on treating hepatic steatosis. A list of DEPs in the liver of steatosis rats by OMT treatment has been identified. Plin2, L-FABP, FASN, and SCD1 are considered the most significantly DEPs. Our data suggest a strong link between OMT and the BP of fatty acid uptake, synthesis, transport, storage, and degradation. Importantly, OMT activates the upstream Sirt1/AMPK signaling in the lipid metabolism regulatory network in the liver. Further studies are required to unravel the interaction, activity regulation, possible complexes and substrates of these proteins, and how they "cross-talk" with PPAR $\alpha$ signaling pathway.

\section{DATA AVAILABILITY STATEMENT}

All datasets generated for this study are included in the article/Supplementary Material.

\section{ETHICS STATEMENT}

All animals received humane care with strict accordance to the criteria outlined in the Guide for the Care and Use of Laboratory Animals. This study was reviewed and approved by the Committee on Animal Research and Ethics of Zhejiang Chinese Medical University (number ZSLL-2014-36).

\section{AUTHOR CONTRIBUTIONS}

HX, G-FC, Y-SM, H-WZ, and DF designed the study. HX, G-FC, Y-SM, G-HL, D-YC, JP, and DF, performed the proteomic analysis and western blotting. HX, G-FC, YZ, Y-HL, XM, and DF performed the animal experiments. HX, G-FC, H-WZ, and DF performed the statistical analyses, interpreted the data, and wrote the manuscript. HX, H-WZ, and DF contributed study materials and consumables. HX, YZ, and DF were responsible for troubleshooting of the experiments, editing of the manuscript, 
and the financial support. HX and G-FC contributed equally to this work. All authors contributed to the final version of the manuscript and approved the final manuscript.

\section{FUNDING}

This work was supported by the National Natural Science Foundation of China (81102707, 81573794, and 81673780), Medical Science and Technology Project of Zhejiang Province (2018KY602), Hangzhou Science and Technology Development Project (20150733Q39 and 20180533B74), and Chinese Medicine Science and Technology Project of Zhejiang Province (2019ZB093).

\section{REFERENCES}

Angeles, T. S., and Hudkins, R. L. (2016). Recent advances in targeting the fatty acid biosynthetic pathway using fatty acid synthase inhibitors. Expert Opin. Drug Discov. 11, 1187-1199. doi: 10.1080/17460441.2016.1245286

Basaranoglu, M., Basaranoglu, G., and Sentürk, H. (2013). From fatty liver to fibrosis: a tale of "second hit". World J. Gastroenterol. 19, 1158-1165. doi: 10.3748/wjg.v19.i8.1158

Buzzetti, E., Pinzani, M., and Tsochatzis, E. A. (2016). The multiple-hit pathogenesis of non-alcoholic fatty liver disease (NAFLD). Metabolism 65, 1038-1048. doi: 10.1016/j.metabol.2015.12.012

Cobbina, E., and Akhlaghi, F. (2017). Non-alcoholic fatty liver disease (NAFLD) -pathogenesis, classification, and effect on drugmetabolizing enzymes and transporters. Drug Metab. Rev. 49, 197-211. doi: 10.1080/03602532.2017. 1293683

Day, E. A., Ford, R. J., and Steinberg, G. R. (2017). AMPK as a therapeutic target for treating metabolic diseases. Trends Endocrinol. Metab. 28, 545-560. doi: 10.1016/j.tem.2017.05.004

Fujii, H., Ikura, Y., Arimoto, J., Sugioka, K., Iezzoni, J. C., Park, S. H., et al. (2009). Expression of perilipin and adipophilin in nonalcoholic fatty liver disease; relevance to oxidative injury and hepatocyte ballooning. J. Atheroscler. Thromb. 16, 893-901. doi: 10.5551/jat.2055

Hsu, W. F., Sheen, L. Y., Lin, H. J., and Chang, H. H. (2016). A review of Western and traditional Chinese medical approaches to managing nonalcoholic fatty liver disease. Evid. Based Complement. Alternat. Med. 2016, 6491420.

Hu, Z., Gu, H., Hu, J., Hu, S., Wang, X., Liu, X., et al. (2018). Quantitative proteomics identify an association between extracellular matrix degradation and immunopathology of genotype VII Newcastle disease virus in the spleen in chickens. J. Proteomics 181, 201-212. doi: 10.1016/j.jprot.2018.04.019

Huang, H., Starodub, O., McIntosh, A., Atshaves, B. P., Woldegiorgis, G., Kier, A. B., et al. (2004). Liver fatty acid-binding protein colocalizes with peroxisome proliferator activated receptor alpha and enhances ligand distribution to nuclei of living cells. Biochemistry 43, 2484-2500. doi: 10.1021/bi0352318

Jensen-Urstad, A. P., and Semenkovich, C. F. (2012). Fatty acid synthase and liver triglyceride metabolism: housekeeper or messenger? Biochim. Biophys. Acta 1821, 747-753. doi: 10.1016/j.bbalip.2011.09.017

Li, L., Liu, Q., Fan, L., Xiao, W., Zhao, L., Wang, Y., et al. (2017). Protective effects of oxymatrine against arsenic trioxide induced liver injury. Oncotarget 8, 12792-12799. doi: 10.18632/oncotarget.12478

Libby, A. E., Bales, E., Orlicky, D. J., and McManaman, J. L. (2016). Perilipin-2 deletion impairs hepatic lipid accumulation by interfering with sterol regulatory element-binding protein (SREBP) activation and altering the hepatic lipidome. J. Biol. Chem. 291, 24231-24246. doi: 10.1074/jbc.m116.759795

Lim, J. W., Dillon, J., and Miller, M. (2014). Proteomic and genomic studies of nonalcoholic fatty liver disease-clues in the pathogenesis. World J. Gastroenterol. 20, 8325-8340. doi: 10.3748/wjg.v20.i26.8325

Liou, C. J., Wei, C. H., Chen, Y. L., Cheng, C. Y., Wang, C. L., and Huang, W. C. (2018). Fisetin protects against hepatic steatosis through regulation of the Sirtl/AMPK and fatty acid $\beta$-oxidation signaling pathway in high-fat

\section{ACKNOWLEDGMENTS}

We thank Dr. Lie-Bo Su from Shanghai Luming Biotech for his assistance with bioinformatics analysis and Dr. XiaoPing Xu from Experimental Animal Research Center, Zhejiang Chinese Medical University for assistance with animal care and maintenance.

\section{SUPPLEMENTARY MATERIAL}

The Supplementary Material for this article can be found online at: https://www.frontiersin.org/articles/10.3389/fphar. 2020.00216/full\#supplementary-material

diet-induced obese mice. Cell Physiol. Biochem. 49, 1870-1884. doi: 10.1159/ 000493650

Liu, Q., Zhu, L., Cheng, C., Hu, Y. Y., and Feng, Q. (2017). Natural active compounds from plant food and Chinese herbal medicine for nonalcoholic fatty liver disease. Curr. Pharm. Des. 23, 5136-5162. doi: 10.2174/ 1381612823666170918120643

Liu, X., Dai, R., Ke, M., Suheryani, I., Meng, W., and Deng, Y. (2017). Differential proteomic analysis of dimethylnitrosamine (DMN)-induced liver fibrosis. Proteomics 17:1700267. doi: 10.1002/pmic.201700267

Lu, M. L., Xiang, X. H., and Xia, S. H. (2016). Potential signaling pathways involved in the clinical application of oxymatrine. Phytother. Res. 30, 1104-1112. doi: $10.1002 /$ ptr.5632

Martin, G. G., Atshaves, B. P., Huang, H., McIntosh, A. L., Williams, B. J., Pai, P. J., et al. (2009). Hepatic phenotype of liver fatty acid binding protein geneablated mice. Am. J. Physiol. Gastrointest. Liver Physiol. 297, G1053-G1065. doi: 10.1152/ajpgi.00116.2009

Martin, G. G., Landrock, D., Chung, S., Dangott, L. J., McIntosh, A. L., Mackie, J. T., et al. (2017). Loss of fatty acid binding protein-1 alters the hepatic endocannabinoid system response to a high fat diet. J. Lipid Res. 58, 2114-2126. doi: 10.1194/jlr.M077891

McIntosh, A. L., Senthivinayagam, S., Moon, K. C., Gupta, S., Lwande, J. S., Murphy, C. C., et al. (2012). Direct interaction of Plin2 with lipids on the surface of lipid droplets: a live cell FRET analysis. Am. J. Physiol. Cell Physiol. 303, C728-C742. doi: 10.1152/ajpcell.00448.2011

McManaman, J. L., Bales, E. S., Orlicky, D. J., Jackman, M., MacLean, P. S., Cain, S., et al. (2013). Perilipin-2-null mice are protected against diet-induced obesity, adipose inflammation, and fatty liver disease. J. Lipid Res. 54, 1346-1359. doi: 10.1194/jlr.M035063

Mukai, T., Egawa, M., Takeuchi, T., Yamashita, H., and Kusudo, T. (2017). Silencing of FABP1 ameliorates hepatic steatosis, inflammation, and oxidative stress in mice with nonalcoholic fatty liver disease. FEBS Open Biol. 7, 1009-1016. doi: 10.1002/2211-5463.12240

Najt, C. P., Senthivinayagam, S., Aljazi, M. B., Fader, K. A., Olenic, S. D., Brock, J. R., et al. (2016). Liver-specific loss of Perilipin 2 alleviates diet-induced hepatic steatosis, inflammation, and fibrosis. Am. J. Physiol. Gastrointest. Liver Physiol. 310, G726-G738. doi: 10.1152/ajpgi.00436.2015

Newberry, E. P., Xie, Y., Kennedy, S. M., Luo, J., and Davidson, N. O. (2006). Protection against Western diet-induced obesity and hepatic steatosis in liver fatty acid-binding protein knockout mice. Hepatology 44, 1191-1205. doi: 10.1002/hep. 21369

Pappachan, J. M., Babu, S., Krishnan, B., and Ravindran, N. C. (2017). Nonalcoholic fatty liver disease: a clinical update. J. Clin. Transl. Hepatol. 5, 384-393. doi: 10.14218/JCTH.2017.00013

Ponugoti, B., Kim, D. H., Xiao, Z., Smith, Z., Miao, J., Zang, M., et al. (2010) SIRT1 deacetylates and inhibits SREBP-1C activity in regulation of hepatic lipid metabolism. J. Biol. Chem. 285, 33959-33970. doi: 10.1074/jbc.M110.12 2978

Purushotham, A., Schug, T. T., Xu, Q., Surapureddi, S., Guo, X., and Li, X. (2009). Hepatocyte-specific deletion of SIRT1 alters fatty acid metabolism and results in 
hepaticsteatosis and inflammation. Cell Metab. 9, 327-338. doi: 10.1016/j.cmet. 2009.02.006

Shi, L., Shi, L., Zhang, H., Hu, Z., Wang, C., Zhang, D., et al. (2013). Oxymatrine ameliorates non-alcoholic fatty liver disease in rats through peroxisome proliferator-activated receptor- $\alpha$ activation. Mol. Med. Rep. 8, 439-445. doi: 10.3892/mmr.2013.1512

Shi, L. J., Shi, L., Song, G. Y., Zhang, H. F., Hu, Z. J., Wang, C., et al. (2013). Oxymatrine attenuates hepatic steatosis in non-alcoholic fatty liver disease rats fed with high fructose diet through inhibition of sterol regulatory element binding transcription factor 1 (Srebf1) and activation of peroxisome proliferator activated receptor alpha (Ppar $\alpha)$. Eur. J. Pharmacol. 714, 89-95. doi: 10.1016/j.ejphar.2013.06.013

Smith, B. K., Marcinko, K., Desjardins, E. M., Lally, J. S., Ford, R. J., and Steinberg, G. R. (2016). Treatment of nonalcoholic fatty liver disease: role of AMPK. Am. J. Physiol. Endocrinol. Metab. 311, E730-E740. doi: 10.1152/ajpendo.00225.2016

Unwin, R. D., Griffiths, J. R., and Whetton, A. D. (2010). Simultaneous analysis of relative protein expression levels across multiple samples using iTRAQ isobaric tags with 2D nano LC-MS/MS. Nat. Protoc. 5, 1574-1582. doi: 10.1038/nprot. 2010.123

Wang, G., Bonkovsky, H. L., de Lemos, A., and Burczynski, F. J. (2015). Recent insights into the biological functions of liver fatty acid binding protein 1. J. Lipid Res. 56, 2238-2247. doi: 10.1194/jlr.R056705

Wolfrum, C., Borrmann, C. M., Borchers, T., and Spener, F. (2001). Fatty acids and hypolipidemic drugs regulate peroxisome proliferator-activated receptors $\alpha$ and $\gamma$-mediated gene expression via liver fatty acid binding protein: a signaling path to the nucleus. Proc. Natl. Acad. Sci. U.S.A. 98, 2323-2328. doi: 10.1073/ pnas.051619898

Wu, S., Wu, F., Ding, Y., Hou, J., Bi, J., and Zhang, Z. (2016). Association of non-alcoholic fatty liver disease with major adverse cardiovascular events: a systematic review and meta-analysis. Sci. Rep. 6:33386. doi: 10.1038/srep33386

Xu, H., Zhou, Y., Liu, Y., Ping, J., Shou, Q., Chen, F., et al. (2016). Metformin improves hepatic IRS2/PI3K/Akt signaling in insulin-resistant rats of NASH and cirrhosis. J. Endocrinol. 229, 133-144. doi: 10.1530/JOE-15-0409

Yao, X., Xia, F., Tang, W., Xiao, C., Yang, M., and Zhou, B. (2018). Isobaric tags for relative and absolute quantitation (iTRAQ)-based proteomics for the investigation of the effect of Hugan Qingzhi on non-alcoholic fatty liver disease in rat. J. Ethnopharmacol. 212, 208-215. doi: 10.1016/j.jep.2017.09.016

Younossi, Z. M., Henry, L., Bush, H., and Mishra, A. (2018). Clinical and economic burden of nonalcoholic fatty Liver disease and nonalcoholic steatohepatitis. Clin. Liver Dis. 22, 1-10. doi: 10.1016/j.cld.2017.08.001

Yu, J., Fan, N., Li, R., Zhuang, L., Xu, Q., and Huang, B. (2019). Proteomic profiling for metabolic pathways involved in interactive effects of elevated carbon dioxide and nitrogen on leaf growth in a perennial grass species. J. Proteome Res. 18, 2446-2457. doi: 10.1021/acs.jproteome.8b00951

Zhang, L., Yao, Z., and Ji, G. (2018). Herbal extracts and natural products in alleviating non-alcoholic fatty liver disease via activating autophagy. Front. Pharmacol. 9:1459. doi: 10.3389/fphar.2018.01459

Zhang, M., Sun, W., Zhou, M., and Tang, Y. (2017). MicroRNA-27a regulates hepatic lipid metabolism and alleviates NAFLD via repressing FAS and SCD1. Sci. Rep. 7:14493. doi: 10.1038/s41598-017-15141-x

Zhang, Y., Geng, C., Liu, X., Li, M., Gao, M., Liu, X., et al. (2016). Celastrol ameliorates liver metabolic damage caused by a high-fat diet through Sirt1. Mol. Metab. 6, 138-147. doi: 10.1016/j.molmet.2016.11.002

Zhao, H. W., Zhang, Z. F., Chai, X., Li, G. Q., Cui, H. R., Wang, H. B., et al. (2016). Oxymatrine attenuates CCl4-induced hepatic fibrosis via modulation of TLR4-dependent inflammatory and TGF- $\beta 1$ signaling pathways. Int. Immunopharmacol. 36, 249-255. doi: 10.1016/j.intimp.2016. 04.040

Conflict of Interest: The authors declare that the research was conducted in the absence of any commercial or financial relationships that could be construed as a potential conflict of interest.

Copyright $\odot 2020 \mathrm{Xu}$, Chen, Ma, Zhang, Zhou, Liu, Chen, Ping, Liu, Mou and Fu. This is an open-access article distributed under the terms of the Creative Commons Attribution License (CC BY). The use, distribution or reproduction in other forums is permitted, provided the original author(s) and the copyright owner(s) are credited and that the original publication in this journal is cited, in accordance with accepted academic practice. No use, distribution or reproduction is permitted which does not comply with these terms. 